\title{
A redox strategy to tailor the release properties of Fe(III)-alginate aerogels for oral drug delivery
}

\author{
Péter Veres ${ }^{\mathrm{a}}$, Dániel Sebők ${ }^{\mathrm{b}}$, Imre Dékány ${ }^{\mathrm{b}}$, Pavel Gurikovc, Irina Smirnovac, István Fábián ${ }^{\mathrm{a}, \mathrm{d}}$, József Kalmár ${ }^{\mathrm{a}, ~ *}$ \\ ${ }^{a}$ Department of Inorganic and Analytical Chemistry, University of Debrecen, Egyetem tér 1, Debrecen, Hungary \\ ${ }^{\mathrm{b}}$ Department of Physical Chemistry and Materials Science, University of Szeged, Rerrich B. tér 1, Szeged, Hungary \\ ${ }^{c}$ Institute for Thermal Separation Process, Hamburg University of Technology (TUHH), Eißendorfer Str. 38, 21073, Hamburg, Germany \\ ${ }^{\mathrm{d}}$ MTA-DE Redox and Homogeneous Catalytic Reaction Mechanisms Research Group, Egyetem tér 1, Debrecen, Hungary
}

\section{A R T I C L E I N F O}

\section{Keywords:}

Drug delivery

Fe(III)-alginate

Aerogel

Redox reaction

\begin{abstract}
A B S T R A C T
Iron(III)-crosslinked alginate aerogel beads $(d=3-5 \mathrm{~mm})$ were prepared and loaded with ibuprofen by using the technique of adsorptive deposition from supercritical $\mathrm{CO}_{2}$. Additional formulations were prepared where the aerogels were co-impregnated by ibuprofen and ascorbic acid. The release of ibuprofen from the Fe(III)-alginate is much faster in $\mathrm{pH}=7.4$ ( $\mathrm{PBS}$ ) than in $\mathrm{pH}=2.0(\mathrm{HCl})$, which can be explained by the faster dissolution and higher swelling of the alginate matrix in PBS. By decreasing the size of the beads and using a higher G content alginate the release rate could be slightly increased. A marked acceleration of drug release was achieved in both $\mathrm{HCl}$ and PBS by incorporating ascorbic acid into the Fe(III)-alginate aerogel preparations. The explanation is that in aqueous media ascorbic acid in situ reduces the crosslinking Fe(III) to Fe(II). The latter does not interact strongly with alginate, which promotes the hydration of the chains, thus the erosion and dissolution of the carrier matrix.
\end{abstract}

\section{Introduction}

Highly porous aerogels can be relatively simply prepared from metal ion crosslinked alginate hydrogels (Garcia-Gonzalez, Alnaief, \& Smirnova, 2011; Quignard, Valentin, \& Di Renzo, 2008; Smirnova \& Gurikov, 2017; Subrahmanyam, Gurikov, Dieringer, Sun, \& Smirnova, 2015; Tkalec, Kranvogl, Uzunalic, Knez, \& Novak, 2016). Similarly to the hydrogels, these aerogels are versatile biomaterials which were recently introduced into the field of drug delivery research (Garcia-Gonzalez et al., 2011; Veronovski, Novak, \& Knez, 2012). The oral (Garcia-Gonzalez, Jin, Gerth, Alvarez-Lorenzo, \& Smirnova, 2015; Mehling, Smirnova, Guenther, \& Neubert, 2009) and mucosal (Goncalves et al., 2016) delivery applications of various active pharmaceutical ingredients, including fat-soluble vitamins (Pantic, Knez, \& Novak, 2016) have been investigated. Layered (Veronovski, Knez, \& Novak, 2013) and core-shell (Ulker \& Erkey, 2014) formulations have also been developed aiming to fine-tune the release kinetics.
The timeframe of the dissolution of adsorbed drugs from alginate aerogel carriers heavily depends on the hydration, swelling and erosion of the matrix. Metal ion alginate gels readily dissolve in neutral or slightly acidic $(\mathrm{pH}>5)$ solutions where the concentration of the cross-linking metal ion is low. In this case some of the coordination bonds break down, metal ions leach from the gel and alginate chains are hydrated. At $\mathrm{pH}<4$ alginate is protonated to alginic acid which forms a barely soluble layer on the surface of the particles limiting the overall rate of the degradation of the matrix (Sriamornsak, Thirawong, \& Korkerd, 2007; Veronovski et al., 2012). Importantly, the kinetics of drug release can be fine-tuned by altering the rate of swelling and erosion of the metal ion alginate matrix.

The erosion rate of alginate gels can be effectively tuned by the use of redox responsive crosslinking agents (Bawa, Pillay, Choonara, \& du Toit, 2009; Li, Maciel, Rodrigues, Shi, \& Tomas, 2015). It was shown that chemically crosslinked alginate hydrogels are sensitive for the reduction of the linking disulfide bonds. The degradation of the hydrogel is triggered by a reductive environment, which induces the release of

\footnotetext{
* Corresponding author.

Email address: kalmar.jozsef@science.unideb.hu (J. Kalmár)
} 
the incorporated active agent (Li et al., 2015; Maciel et al., 2013). Another promising strategy to produce redox responsive hydrogels is to crosslink alginate chains by a redox active metal ion which radically changes its affinity towards alginate when its oxidation state changes. The most successful systems are based on the Fe(III)/Fe(II) pair. Iron(III) interacts strongly with alginate (Dong, Dong, Cao, Han, \& Ding, 2011; Lee, Min, \& Kim, 1996), while Fe(II) is not capable of crosslinking the chains in aqueous solution (Bruchet \& Melman, 2015; M. Giammanco, Sosnofsky, \& Ostrowski, 2015; Jin et al., 2012). Thus by the in situ reduction of Fe(III) to Fe(II), the degradation of the ionically crosslinked hydrogel is triggered and the active ingredient is released. Three different approaches are described in the literature to utilize the Fe(III)/ $\mathrm{Fe}(\mathrm{II})$ system. The electrochemical approach is based on the deposition of Fe(III)-alginate hydrogel on the surface of an electrode, where an independent signal induces the electrochemical reduction of $\mathrm{Fe}(\mathrm{III})$ (Jin et al., 2012; Katz et al., 2015). The photochemical approach is based on the application of a photoactive reducing agent (e.g. lactic acid) which can reduce Fe(III) only under illumination (Bruchet, Mendelson, \& Melman, 2013; Giammanco et al., 2015; Narayanan, Melman, Letourneau, Mendelson, \& Melman, 2012). Finally, Fe(III)-alginate hydrogel degradation can be triggered by the addition of a strong reducing agent (e.g. ascorbic acid) under controlled conditions (Bruchet \& Melman, 2015).

In this study we shown that the drug delivery properties of dry Fe(III)-alginate aerogels can be tuned by incorporating ascorbic acid into the formulations. Fe(III)-alginate aerogels were synthesized by the sol-gel method in the form of spherical particles $(d=3-5 \mathrm{~mm})$ and loaded with the model drug ibuprofen in supercritical $\mathrm{CO}_{2}$. Some of the ibuprofen impregnated $\mathrm{Fe}(\mathrm{III})$-alginate aerogels were co-impregnated with ascorbic acid. Finally, in vitro dissolution experiments were performed to show that the rate of drug release is significantly faster in the case of the latter system, as ascorbic acid in situ reduces Fe(III) to Fe(II) upon hydration of the aerogels.

\section{Experimental}

\subsection{Materials}

Two different Na-alginates with high guluronic acid content (high G, HG) and low guluronic acid content (low G, LG) from FMC Biopolymer (Norway) were used. Their detailed characterization is given elsewhere (Agulhon, Robitzer, Habas, \& Quignard, 2014). The G/M ration is $30 / 70$ in the low $\mathrm{G}$ alginate and that is $70 / 30$ in the high $\mathrm{G}$ alginate. $\mathrm{FeCl}_{3} \times 6 \mathrm{H}_{2} \mathrm{O}$, ibuprofen [2-(4-(2-methyl-propyl)phenyl) propionic acid] and ascorbic acid were purchased from Sigma-Aldrich. Supercritical $\mathrm{CO}_{2}$ was produced from $99.95 \%$ pure gas (AGA Gas $\mathrm{GmbH}$, Germany). All aqueous solutions were prepared with Milli-Q water (Millipore). Other chemicals $\left(\mathrm{HCl}, \mathrm{NaOH}\right.$, and $\mathrm{NaH}_{2} \mathrm{PO}_{4}$ ) were ACS reagent grade (Sigma-Aldrich).

\subsection{Synthesis of $\mathrm{Fe}(\mathrm{III})$-alginate aerogel beads}

Spherical alginate beads $(3.0-4.5 \mathrm{~mm})$ were synthesized using the following method (Veronovski et al., 2013). The $2 \mathrm{w} / \mathrm{w} \%$ aqueous solution of either the low $\mathrm{G}$ or the high $\mathrm{G}$ alginate was dropped into $0.05 \mathrm{M}$ $\mathrm{FeCl}_{3}$ solution (gelation bath) from fixed height of $10 \mathrm{~cm}$. The $\mathrm{FeCl}_{3}$ solution was slightly acidified previously with $\mathrm{HCl}$ to avoid the hydrolysis of $\mathrm{Fe}^{3+}$. In order to achieve the homogeneous size distribution of the alginate droplets the alginate solutions were extruded through a plastic tube of $d=2 \mathrm{~mm}$. Another batch of droplets was produced from both alginates implementing a needle at the end of the tube to obtain smaller gel spheres. During the preparation a moderate stirring (ca. 200 rpm) of the gelation bath was used to ensure the formation of spherical beads and to prevent the agglomeration and sedimentation of the droplets. After gelation, the beads were placed into a fresh $0.05 \mathrm{M} \mathrm{FeCl}_{3}$ solution for $24 \mathrm{~h}$. This was followed by multiple step solvent exchange. The alginate beads were placed for $24 \mathrm{~h}$ into $30 \mathrm{~V} / \mathrm{V} \%, 60 \mathrm{~V} / \mathrm{V} \%, 90 \mathrm{~V} / \mathrm{V} \%$ ethanol-water mixtures and two times into pure ethanol. After the last step, the ethanol content of the soaking liquid was checked by measuring its density with a density meter (DMA 4500, Anton Paar Company, Austria). The drying procedure was initiated only when the ethanol content reached min. $98.5 \mathrm{~V} / \mathrm{V} \%$ to ensure single phase conditions during subsequent supercritical drying (Subrahmanyam et al., 2015). The samples were dried with supercritical $\mathrm{CO}_{2}$ at $45^{\circ} \mathrm{C}$ and 140 bar using a continuous flow process in a high pressure autoclave, as described elsewhere (Goncalves et al., 2016; Smirnova, Mamic, \& Arlt, 2003). Altogether, four different aerogel samples were produced as summarized in Table 1.

Low-temperature supercritical drying in $\mathrm{CO}_{2}$ was found to be optimal method for the production of dry, crack-free Fe(III)-alginate aerogel particles that retain the structure of the parent hydrogels (De Cicco et al., 2016; Tkalec et al., 2016; Zhao, Malfait, Guerrero Alburquerque, Koebel, \& Nystrom, 2018). No other drying method ensures the good mechanical properties of the product accompanied by a high porosity and a dominantly mesoporous, interconnected pore network.

The Fe-content of the alginate samples was measured by the following method. The aerogel was dissolved in a $0.1 \mathrm{M}$ EDTA solution at neutral $\mathrm{pH}$. Water was evaporated and the dry, solid residue was digested with concentrated $\mathrm{HNO}_{3}$. The Fe-content of this solution was measured by ICP-AES (Agilent Technologies ICP-OES SVDV 5100) after dilution.

\subsection{Impregnation of $\mathrm{Fe}(\mathrm{III})$-alginate aerogels}

In the case of aerogels, the most efficient loading process developed for the encapsulation of nonpolar drugs is the adsorptive deposition of the drug from supercritical $\mathrm{CO}_{2}$. This process is analogous to the traditional impregnation from organic solvents. Importantly, the porous matrix remains intact upon the removal of sc. $\mathrm{CO}_{2}$ after impregnation, because no capillary stress forces are present in the system. This tech-

Table 1

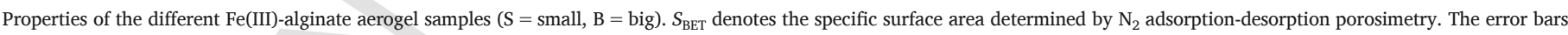
were calculated from parallel measurements $(n=3$, for porosimetry $n=2)$.

\begin{tabular}{|c|c|c|c|c|c|}
\hline Sample & $\mathrm{G}$ content $(\mathrm{w} / \mathrm{w} \%)^{\mathrm{a}}$ & $\mathrm{Fe}(\mathrm{III})$ content $(\mathrm{w} / \mathrm{w} \%)$ & bead diameter $(\mathrm{mm})$ & $S_{\mathrm{BET}}\left(\mathrm{m}^{2} / \mathrm{g}\right)$ & Total pore volume $\left(\mathrm{cm}^{3} / \mathrm{g}\right)$ \\
\hline S-LG & 30 & $8.56 \pm 0.04$ & $3.0 \pm 0.2$ & $420 \pm 19$ & $1.2 \pm 0.2$ \\
\hline B-LG & 30 & $8.08 \pm 0.03$ & $4.5 \pm 0.3$ & $406 \pm 18$ & $1.8 \pm 0.2$ \\
\hline S-HG & 70 & $6.99 \pm 0.05$ & $3.3 \pm 0.2$ & $442 \pm 25$ & $1.8 \pm 0.2$ \\
\hline B-HG & 70 & $6.82 \pm 0.02$ & $4.5 \pm 0.3$ & $316 \pm 19$ & $1.2 \pm 0.2$ \\
\hline
\end{tabular}

a Data taken from ref. (Agulhon et al., 2014). 
nique results in reasonably high loadings and often leads to the amorphization of the drugs inside the pores of the carrier (Gurikov \& Smirnova, 2017). This technique is regarded to be optimal only if the aerogel is saturated with the drug in supercritical $\mathrm{CO}_{2}$, thus the active ingredient is often used in excess.

In one set of experiments the Fe(III)-alginate aerogel beads were impregnated with ibuprofen alone, in another set of experiments the beads were simultaneously impregnated with ibuprofen and ascorbic acid. Weighted amounts of aerogel beads and active ingredients were wrapped separately in filter paper and placed into a $250 \mathrm{~mL}$ autoclave. Ibuprofen and ascorbic acid were taken in excess to guarantee the highest possible loadings at the experimental conditions, i.e. the concentrations of the components in sc. $\mathrm{CO}_{2}$ were at their solubility limits. The vessel was warmed up to $45^{\circ} \mathrm{C}$ and sc- $\mathrm{CO}_{2}$ was pumped into it until the pressure reached $200 \mathrm{bar}$. These conditions were kept constant for $6 \mathrm{~h}$. The pressure was released in two steps. First, a 120 bar pressure drop was carried out in $10 \mathrm{~s}$, and from there the pressure was released at ca. 3-4bar/min. After the depressurization the loaded aerogel samples were removed from the vessel.

The amount of the adsorbed ibuprofen and ascorbic acid were determined by UV-vis spectrophotometry after soaking the loaded aerogel samples in methanol. Ibuprofen was measured at $220 \mathrm{~nm}$ and ascorbic acid at $245 \mathrm{~nm}$. Iron(III) salts do not dissolve in methanol and thus did not interfere with the measurements. All measurements were run in triplicates. The loadings of the samples are summarized in Table 2.

\subsection{Aerogel characterization}

\subsection{1. $\mathrm{N}_{2}$ adsorption-desorption porosimetry}

The specific surface area, pore volume and pore size distribution of aerogel samples were measured after degassing at $40^{\circ} \mathrm{C}$ for $24 \mathrm{~h}$ by $\mathrm{N}_{2}$ adsorption-desorption porosimetry using a Quantachrome Nova 2000e instrument. Specific surface area $\left(S_{\mathrm{BET}}\right)$ was obtained by the multipoint BET method. Pore size distribution was calculated from the desorption branch using the BJH method. All measurements were duplicated and the difference in $S_{\mathrm{BET}}$ and pore size distribution between parallel runs were found to be not more than $10 \%$.

\subsubsection{Scanning electron microscopy}

Scanning electron microscopic (SEM) images were taken by a Leo 1530 microscope (Carl Zeiss, Germany). Samples were immobilized on

\section{Table 2}

Drug loading of the different Fe(III)-alginate aerogel samples ( $=$ loaded with ibuprofen only, IA = loaded with ibuprofen and ascorbic acid). Properties of the pristine aerogel samples are given in Table 1. The error bars were calculated from parallel measurements $(n=3)$.

\begin{tabular}{llll}
\hline Sample & $\begin{array}{l}\text { Ibuprofen mass } \\
\text { content }(\mathrm{w} /\end{array}$ & $\begin{array}{l}\text { Ascorbic acid } \\
\text { mass content } \\
(\mathrm{w} / \mathrm{w} \%)\end{array}$ & $\begin{array}{l}\text { Specific loading }(\mathrm{mg} \\
\text { ibuprofen } / \mathrm{m}^{2} \\
\text { aerogel })\end{array}$ \\
\hline S-LG-I & $41 \pm 4$ & - & $1.5 \pm 0.1$ \\
B-LG-I & $37.9 \pm 0.2$ & - & $1.50 \pm 0.01$ \\
S-HG-I & $36.4 \pm 0.9$ & - & $1.30 \pm 0.02$ \\
B-HG-I & $37 \pm 2$ & - & $1.8 \pm 0.1$ \\
S-LG- & $34 \pm 5$ & $4.0 \pm 0.4$ & $1.2 \pm 0.1$ \\
IA & $35 \pm 2$ & $4.3 \pm 0.7$ & $1.32 \pm 0.05$ \\
B-LG- & $33.6 \pm 0.9$ & $3.4 \pm 0.4$ & $1.14 \pm 0.02$ \\
IA & $34 \pm 3$ & $3.4 \pm 0.4$ & $1.6 \pm 0.1$ \\
S-HG- & & & \\
IA & & & \\
B-HG- & & & \\
IA & & &
\end{tabular}

carbon tapes and sputtered with gold $(7 \mathrm{~nm})$. Pictures were taken at an accelerating voltage of $5 \mathrm{kV}$ and a working distances of $7.4 \mathrm{~mm}$.

\subsubsection{Infrared spectroscopy}

Infrared spectra of the raw aerogels, the crystalline active compounds and the loaded aerogels were measured with FT-IR technique on a Jasco FT/IR-4100 spectrometer using $\mathrm{KBr}$ discs.

\subsubsection{Small-angle X-ray scattering (SAXS)}

SAXS curves were recorded with a slit-collimated Kratky compact small-angle system (KCEC/3 Anton-Paar KG, Graz, Austria) equipped with a position-sensitive detector (PSD $50 \mathrm{M}$ from M. Braun AG Munich, Germany) containing 1024 channels $55 \mu \mathrm{m}$ in width. $\mathrm{CuK}_{\alpha}$ radiation was generated by a Philips PW1830 X-ray generator operating at $40 \mathrm{kV}$ and $30 \mathrm{~mA}$. The surface area per unit volume $(S / V)$ and the specific surface area $\left(a^{s}\right)$ values were determined by using Eqs. (1) and (2).

$$
S / V=4 w_{1} w_{2} K_{P} / Q
$$

$a^{s}=10^{3} \rho_{a p p}{ }^{-1} S / V$

where $Q$ is the invariant and $\rho_{a p p}$ is the apparent density, while $w_{1}$ and $w_{2}$ are the volume fractions of the solid phase and pores, respectively. $K_{p}, Q$ and $L_{c}$ (correlation length - the average intersection length of the solid phase) were determined according to Eqs. (3)-(5).

$$
\begin{aligned}
& K_{P}=\lim _{h \rightarrow \infty} I(h) h^{3} \\
& Q=\int_{0}^{\infty} I(h) h d h \\
& L_{c}=\pi \int_{0}^{\infty} I(h) d h / Q
\end{aligned}
$$

The scattering vector $(h)$ was defined as $h=4 \pi \sin \theta \lambda^{-1}$, where $\theta$ is one-half of the scattering angle (Glatter \& Kratky, 1982; Jánosi, 1986; Porod, 1951). The compactness (mass fractal) properties of the materials were characterized by the slope $(p)$ of the fitted curve in log-log (fractal) representation. The mass fractal dimension $\left(D_{m}\right)$ of the two-phase system can be determined by using Eq. (6):

$$
I(h)=I_{0} h^{-\mathrm{p}}=I_{0} h^{-\mathrm{D}_{m}}
$$

The SAXS measurements were performed in a slit collimation geometry, where no desmearing correction was applied, therefore the aforementioned relationships for $D_{m}, K_{p}$ and $Q$ refer to slit-smeared scattering curves.

\subsubsection{X-ray diffraction (XRD)}

The X-ray diffractograms of the pristine and loaded aerogels, the drugs and the physical mixtures of pristine aerogels and drugs were registered on a Philips X-ray diffractometer with $\mathrm{CuK}_{\alpha}(=0.1542 \mathrm{~nm})$ as the radiation source. Data was recorded at ambient temperature in the $2-40^{\circ}(2 \Theta)$ range with a step size of $0.02^{\circ}(2 \Theta)$. 


\subsection{Drug release measurements}

Drug release was followed in $\mathrm{HCl}(\mathrm{pH}=2.0)$ and in phosphate buffered saline solution (PBS, $\mathrm{pH}=7.4$ ) using on-line UV-vis detection (Veres et al., 2017). The $\mathrm{pH}$ values of the solutions were set to either 2.00 or 7.40 using a Metrohm 785 DMP Titrino automatic titrator with a $6.0262 .100 \mathrm{pH}$-electrode. The uncertainty of the $\mathrm{pH}$ measurement was \pm 0.01 unit.

Loaded aerogel beads (1-2 pieces) were weighted with $0.01 \mathrm{mg}$ precision into a carefully dried spectrophotometric cuvette. The total weight of the samples was $1.0-1.5 \mathrm{mg}$ in the case of the smaller beads and ca. $3.0-3.5 \mathrm{mg}$ in the case of the larger beads. The total amount of ibuprofen introduced into release media was therefore $0.1-0.4 \mathrm{mg}$ / $\mathrm{mL}$. The cuvette was placed into the cell of a custom built fiber optic $\mathrm{UV}$-vis spectrophotometer equipped with a fast CCD detector (Avantes) (Ditroi et al., 2016) and thermostated at $37.0 \pm 0.1^{\circ} \mathrm{C}$. On-line detection was started, and $3.0 \mathrm{~mL}$ pre-heated release medium (pH $2.0 \mathrm{HCl}$ solution or pH 7.4 PBS) was injected into the cuvette. A perforated PTFE placeholder was used to fix the position of the aerogel beads during the whole experiment and thus minimalize the chance of any disturbing effect of the movement of the spheres in front of the light beam. During the injection and under the whole length of the release experiment the suspension was stirred by a $2 \times 8 \mathrm{~mm}$ PTFE coated magnetic stirbar at 300 $\mathrm{rpm}$. The detector was typically operated with $30 \mathrm{~ms}$ integration time and 20 subsequent spectra were averaged. Absorbance change was followed in the $200-500 \mathrm{~nm}$ wavelength range with $1.0 \mathrm{~nm}$ steps for $6200 \mathrm{~s}$ with a time resolution of $12 \mathrm{~s}$. As the drug was released from the aerogel, the characteristic UV-vis spectrum of ibuprofen (200-300 nm) was detected in the suspension. All measurements were run in duplicates, and the mean values are displayed in the corresponding figures. Maximum and minimum values are also shown in representative curves to demonstrate the high reproducibility of the measurements (Figure S1 in the Supporting Information). (Rousseeuw \& Verboven, 2002).

It should be noted that the alginate matrices dissolved simultaneously with the release of the drug. Thus, independent dissolution experiments were performed with unloaded aerogel samples under the conditions of the drug release tests. Aqueous alginate absorbs light in the 200-350 nm region.

The release curves (amount of dissolved ibuprofen versus time) were calculated from the time-resolved absorbance signal using the molar absorbance of the drug. The contribution of dissolved alginate to the absorbance signal was taken into account at every time-point by using the dual wavelength method developed by Liu and Zhu (Liu, Zhu, \& Chai, 2011; Wang, Zhu, Hunt, \& Zhan, 2012). First, the spectrum of aqueous alginate was measured under the applied conditions (in $\mathrm{HCl}$ or PBS). Second, a reference wavelength was chosen where dissolved ibuprofen has zero absorbance $(300 \mathrm{~nm})$, and the spectrum of alginate was reconstructed from this reference absorbance by using the known molar absorbances in the whole wavelength range. Finally, the spectrum of alginate was subtracted from the recorded UV-vis spectrum. The remaining signal is exclusively attributed to dissolved ibuprofen.

The in situ formation of $\mathrm{Fe}(\mathrm{II})$ during the dissolution tests was demonstrated by running the experiments under $\mathrm{O}_{2}$ free conditions in a Schlenk-line and adding terpyridine $\left(2,2^{\prime}: 6^{\prime}, 2^{\prime \prime}\right.$-terpyridine $)$ to the final solutions after $30 \mathrm{~min}$. An intensive red coloration was observed in the case of ascorbic acid loaded aerogels indicating the presence of aqueous $\mathrm{Fe}(\mathrm{II})$.

\section{Results and discussion}

Both the loaded and the unloaded aerogel beads are dry, yellowish solids and display a smooth, homogeneous texture for the naked eye (see pictures in Figure S2 in the Supporting Information).

\subsection{Scanning electron microscopy}

Representative SEM images of pristine and loaded alginate aerogels in $60 \mathrm{k} \times$ magnification are shown in Figs. 1 and S3 in the Supporting Information. All samples display a similar open meso- and macroporous, sponge-like structure that is characteristic to alginate aerogels (Pantic et al., 2016; Robitzer, David, Rochas, Di Renzo, \& Quignard, 2008a, 2008b). No major alterations can be discovered between the structures of the pristine and the loaded samples.

\section{2. $N_{2}$ adsorption-desorption porosimetry}

The $\mathrm{N}_{2}$ adsorption-desorption isotherms (Fig. 2) of the Fe(III)-alginate aerogels are type IV hysteresis curves, characteristic for mesoporous materials with macropores. The position of the hysteresis loop indicates a very wide pore size distribution with a large number of macropores which can also be seen on the SEM micrographs. The pore size distribution curves of the samples were calculated from the desorption branches of the isotherms using the BJH method (Fig. 2). The resulting distribution curves are very wide with a maximum around $30 \mathrm{~nm}$ in diameter. It is evident from the SEM pictures that a significant fraction of the pores are larger than $200 \mathrm{~nm}$. However, $\mathrm{N}_{2}$ porosimetry is not suitable for the characterization of these large cavities.

The specific surface area $\left(S_{\mathrm{BET}}\right)$ and the total pore volume of the freshly synthesized beads vary between 320 and $440 \mathrm{~m}^{2} / \mathrm{g}$ and $1.20-1.77 \mathrm{~cm}^{3} / \mathrm{g}$, respectively (Table 1 ). These values are in good agreement with previously reported results (Alnaief, Alzaitoun, Garcia-Gonzalez, \& Smirnova, 2011; Garcia-Gonzalez et al., 2011; Veronovski et al., 2013). It should be noted, that the alginate matrix is sensitive to storage conditions (e.g. humidity, UV radiation). After storing the pristine Fe(III)-alginate gels for 1year in a closed plastic vessel, the color

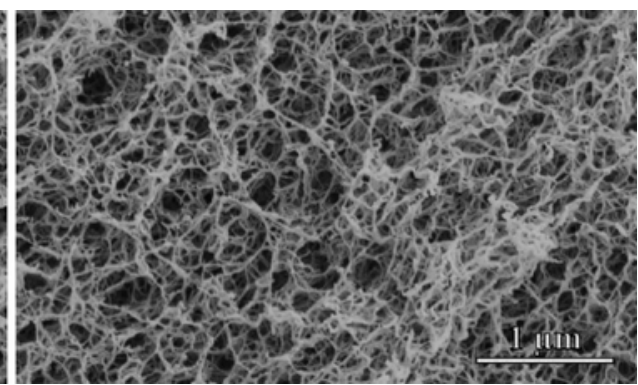

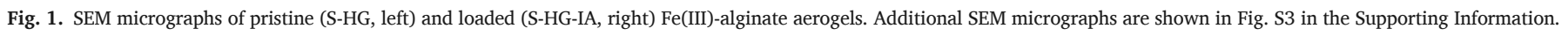



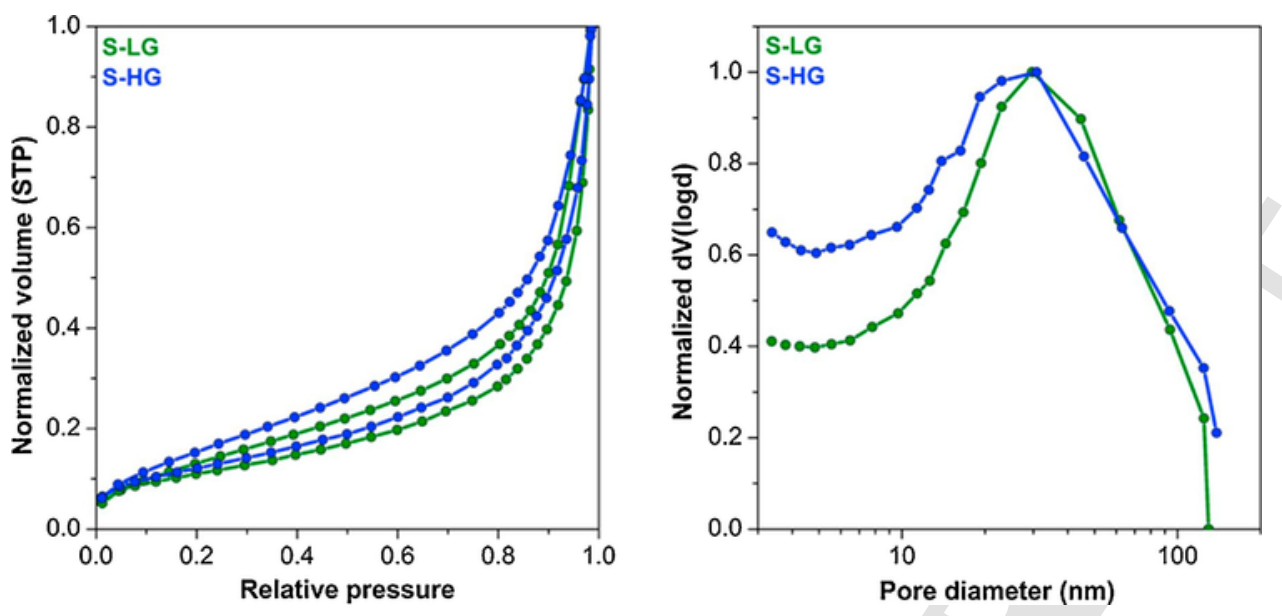

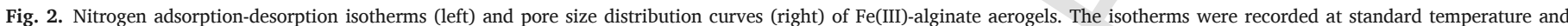
pressure. Pore size distributions were calculated from desorption curves by the BJH method.

of the beads changed from yellow to brownish (most likely due to oxidation of the alginate backbone). The fine structure of the matrices also changed according to $\mathrm{N}_{2}$ porosimetry, especially in the case of the high G alginate aerogels. The $S_{\mathrm{BET}}$ of low G gels decreased from 400 to $420 \mathrm{~m}^{2} / \mathrm{g}$ to 300 to $320 \mathrm{~m}^{2} / \mathrm{g}$ and that of the high $\mathrm{G}$ gels decreased from 320 to $440 \mathrm{~m}^{2} / \mathrm{g}$ to 240 to $270 \mathrm{~m}^{2} / \mathrm{g}$. The pore size distribution of the aerogels did not change in the $d=4-200 \mathrm{~nm}$ mesopore range. Surprisingly, the total pore volume of the high $\mathrm{G}$ alginate aerogels increased significantly from $1.20-1.70 \mathrm{~cm}^{3} / \mathrm{g}$ to $7.20-8.30 \mathrm{~cm}^{3} / \mathrm{g}$. A possible explanation for the structural changes is the rearrangement of the coordination sphere of the Fe(III) ions, i.e. the formation of Fe(III)-hydroxides and other hydrated salts. This could cause the undesirable deformation of the backbone of the aerogels.

\subsection{Infrared spectroscopy}

The representative FT-IR spectra of pristine (S-LG) and loaded (S-LG-I) aerogel samples are shown in Fig. 3, together with that of crystalline ibuprofen. In the spectrum of the pristine $\mathrm{Fe}(\mathrm{III})$-alginate aerogel characteristic bands can be observed for the asymmetric stretching vibrations of aliphatic $\mathrm{C}-\mathrm{H}$ bonds $\left(3424 \mathrm{~cm}^{-1}\right)$, the stretching vibrations of $\mathrm{O}-\mathrm{H}$ bonds $\left(2920 \mathrm{~cm}^{-1}\right)$, the asymmetric stretching of $\mathrm{COO}-$ $\left(1734 \mathrm{~cm}^{-1}\right)$ and the stretching of $\mathrm{C}=\mathrm{O}\left(1410 \mathrm{~cm}^{-1}\right)$ and $\mathrm{C}=\mathrm{C}$ bonds $\left(1030 \mathrm{~cm}^{-1}\right)$ (Daemi \& Barikani, 2012; van Hoogmoed, Busscher, \& de Vos, 2003).

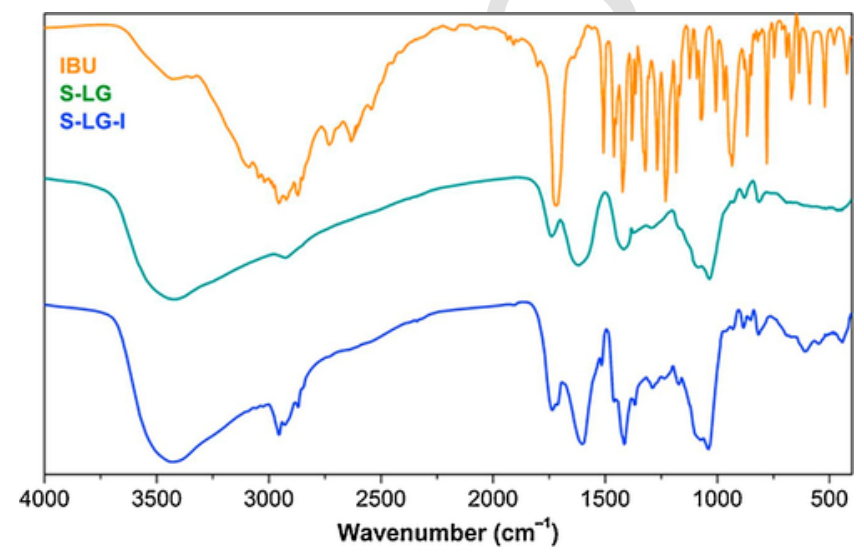

Fig. 3. Representative IR spectra of a pristine Fe(III)-alginate aerogel (S-LG), ibuprofen and an ibuprofen impregnated aerogel (S-LG-I).
The characteristic bands of ibuprofen can be detected in superposition to those of the aerogel backbone in the impregnated samples, confirming the successful loading. The new bands in the spectrum of S-LG-I between 3090 and $2850 \mathrm{~cm}^{-1}$ are related to the asymmetric stretching of aliphatic and aromatic $\mathrm{C}-\mathrm{H}$ bonds of ibuprofen. The band originating from the $\mathrm{C}=\mathrm{O}$ stretching of the new carboxylic group is seen at $1710 \mathrm{~cm}^{-1}$. The $\mathrm{C}=\mathrm{C}$ ring vibration causes a lower intensity brand at $1515 \mathrm{~cm}^{-1}$.

The presence of ascorbic acid does not alter the IR spectra of those aerogel samples which contain ascorbic acid together with ibuprofen, because the loading of ascorbic acid is 1 magnitude lower than that of ibuprofen.

\subsection{X-ray diffraction $(X R D)$}

The XRD patterns of crystalline ibuprofen and ascorbic acid are shown in Fig. 4. The XRD patterns of the impregnated L-LG-I and L-LG-IA aerogel samples are given in the same figure. The absence of the characteristic diffraction peaks in the case of the loaded gels clearly indicate that both ibuprofen and ascorbic acid are present in amorphous states in these samples. It is generally true that the adsorptive deposition (or so called adsorptive precipitation) of active ingredients from solutions in supercritical $\mathrm{CO}_{2}$ into the pores of aerogels results in non-crystalline solid adsorbates, which greatly enhances the dissolution rate and bioavailability of these ingredients (Gurikov \& Smirnova, 2017).

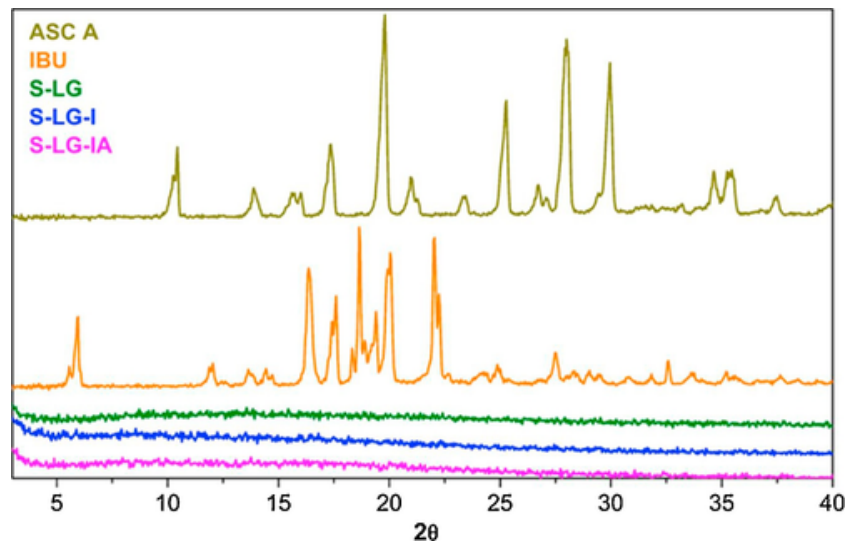

Fig. 4. Representative XRD patterns of crystalline ibuprofen and ascorbic acid, and the impregnated Fe(III)-aerogels S-LG-I and S-LG-IA containing only ibuprofen or both of the active ingredients, respectively. 


\subsection{Small-angle $X$-ray scattering (SAXS)}

The fine-structure of all Fe(III)-alginate aerogel samples could clearly be determined by SAXS. When representing the scattering curves in $h^{2} \cdot I(q)$ vs. $h$ (Kratky-plot, shown in Figs. 5 and in S4 in the Supporting Information) a peak should be seen at $h \approx 1 / R_{c}$ in the case of structures containing randomly oriented rods (where $R_{c}$ is the mean gyration radius of the rods). This peak is absent in the Fe(III)-alginate aerogels indicating the so-called multiple junction structure (Agulhon, Robitzer, David, \& Quignard, 2012). The formation of the multiple junction structure takes place when the cross-linking and gelation of alginate is carried out under acidic conditions. In our case, the Fe(III) solutions used in the synthesis were indeed slightly acidified to avoid the hydrolysis of the salt.

In addition to the structure identification, several structural parameters can be calculated from the $\log$ - $\log$ (fractal) and Porod-plots of the scattering curves (Figs. 5 and S4 in the Supporting Information).
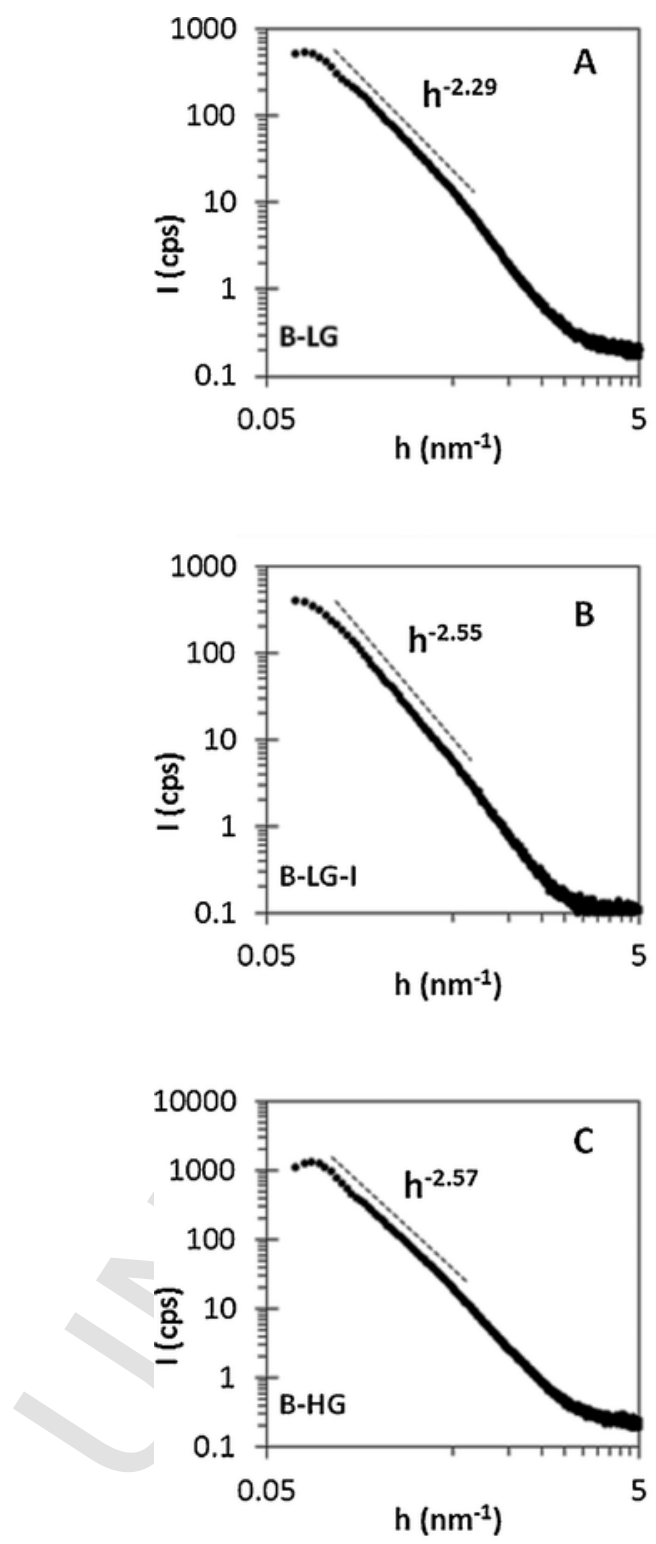

The mass fractal dimension $\left(D_{m}\right)$ is indicative of the compactness of a material. The value of $D_{m}$ may vary from 2 for porous structures to 3 for compact structures. According to mass fractal values (Table 3), it can be established that the pristine low-G samples (S-LG and B-LG) have more porous structures than the pristine high-G aerogels (S-HG and B-HG). Furthermore, a very clear trend can be observed within a series of samples before and after impregnation. B-HG, B-HG-I and BH-IA have systematically increasing $D_{m}$ values $(2.29,2.55$ and 2.64 , respectively) which obviously indicates that the porous system becomes more and more compact with increasing loading. This is also confirmed by the increase in the correlation length $\left(L_{\mathrm{c}}\right)$, i.e. the average intersection length of the solid phase of the same samples $(9.9,11.8$ and $12.5 \mathrm{~nm}$, respectively).

The surface area per unit volume $(S / V)$ and the specific surface area $\left(a^{s}\right)$ values can be calculated as described by Eqs. (1) and (2) when the so-called Porod constant $\left(K_{p}\right)$ and invariant $(Q)$ are determined (Eqs. (3) and (4)). The $S / V$ values are primary experimental data, and they describe the ratio of all surfaces and total volumes in two-phase sys-
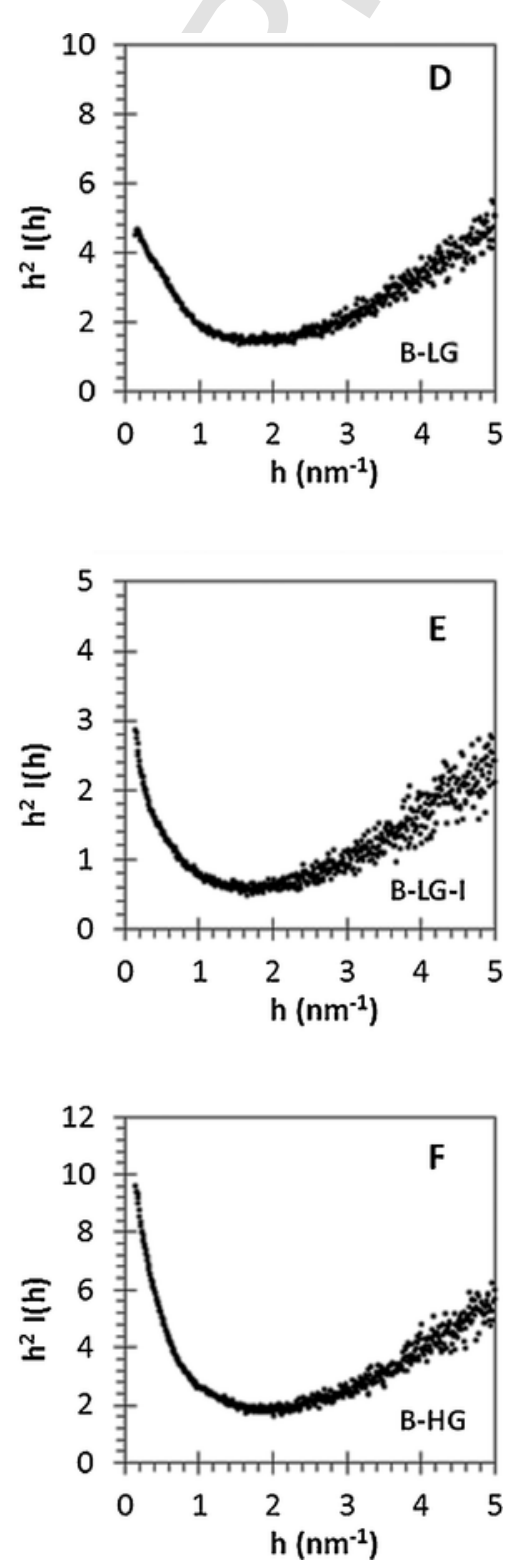

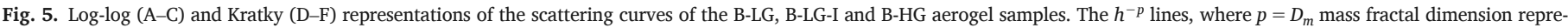

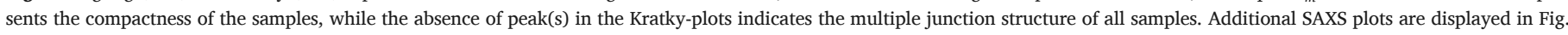
S4 in the Supporting Information. 
Table 3

The mass fractal dimension $\left(D_{\mathrm{m}}\right)$, the correlation length $\left(L_{\mathrm{c}}\right)$, the surface area per unit volume $(S / V)$ and the specific surface area ( $\left.a_{\text {SAXS }}^{s}\right)$ values of Fe(III)-alginate aerogel samples. The error bars represent the combined uncertainty arising from the experimental error and data fitting.

\begin{tabular}{lllll}
\hline Sample & $D_{\mathrm{m}}$ & $L_{\mathrm{c}}(\mathrm{nm})$ & $\begin{array}{l}S / V \\
\left(\mathrm{~m}^{2} / \mathrm{cm}^{3}\right)\end{array}$ & $a_{\text {SAXS }}^{\mathrm{s}}\left(\mathrm{m}^{2} / \mathrm{g}\right)$ \\
\hline S-LG & $2.4 \pm 0.1$ & $10.8 \pm 0.5$ & $31 \pm 2$ & $669 \pm 30$ \\
B-LG & $2.3 \pm 0.1$ & $9.9 \pm 0.4$ & $30 \pm 2$ & $560 \pm 28$ \\
B-LG-I & $2.6 \pm 0.1$ & $11.8 \pm 0.6$ & $22 \pm 1$ & $484 \pm 20$ \\
B-LG-IA & $2.6 \pm 0.1$ & $12.5 \pm 0.5$ & $23 \pm 1$ & $456 \pm 23$ \\
S-HG & $2.5 \pm 0.1$ & $10.7 \pm 0.5$ & $23 \pm 1$ & $479 \pm 24$ \\
B-HG & $2.6 \pm 0.1$ & $12.2 \pm 0.6$ & $24 \pm 1$ & $541 \pm 29$ \\
\hline
\end{tabular}

tems (Table 3). If the apparent density $\left(\rho_{\text {app }}\right.$ ) is known, the specific surface area $\left(a_{\text {SAXS }}^{s}\right)$ can be calculated. As seen in Table 3, the $a_{\text {SAXS }}^{s}$ values (ca. $5-600 \mathrm{~m}^{2} / \mathrm{g}$ ) are higher than the $S_{B E T}$ values (ca. $3-400 \mathrm{~m}^{2} / \mathrm{g}$, see Table 1.). The explanation for this difference is that the contribution of the macropores larger than $d=200 \mathrm{~nm}$ are ignored by $\mathrm{N}_{2}$ porosimetry, but the $a_{S A X S}^{s}$ is directly calculated from surface area per unit volume $(S / V)$ data.

\subsection{Drug release experiments}

When the Fe(III)-alginate aerogels were impregnated with ibuprofen alone, a drug loading of $36-41 \mathrm{w} / \mathrm{w} \%$ was achieved (Table 2). Simultaneous impregnation with ibuprofen and ascorbic acid resulted in an ibuprofen loading of 34-35w/w\%, and the absorbed amount of absorbed ascorbic acid was in the range of $3.4-4.3 \mathrm{w} / \mathrm{w} \%$. A simple calculation yields that approximately the same molar amount of active substance was absorbed cumulatively in each process.

The release of ibuprofen was measured at $\mathrm{pH}=2.0$ in $\mathrm{HCl}$ and at $\mathrm{pH}=7.4$ in PBS. All of the different Fe(III)-alginate matrices dissolved (at least partially) in both media in the timeframe of the release experiments. Because the dissolution of the carrier matrix has a marked effect on the release of the adsorbed drug, independent tests were performed to study this phenomenon. Weight-normalized alginate dissolution curves recorded in $\mathrm{HCl}$ and in PBS are shown in Fig. 6. The beads completely dissolved in $2 \mathrm{~h}$ in PBS, but not in $\mathrm{HCl}$. In $\mathrm{HCl}$ neither the size of beads, nor the composition of the backbone affects the rate of dissolution. Earlier studies with $\mathrm{Ca}(\mathrm{II})$-alginate hydrogels and aerogels concluded that alginate particles form a macroscopic semi-permeable outer crust in HCl (Sriamornsak et al., 2007; Veronovski et al., 2012). This layer is composed of alginic acid (so called acid gel) which has a low solubility in water. The alginic acid layer never dissolves completely as it is replenished immediately from alginate in contact with $\mathrm{HCl}$, limiting the overall rate of dissolution of metal ion alginate particles in $\mathrm{HCl}$. This mechanism is practically independent of the alginate bead size and the composition of the polymer, therefore can be operative also in the case of the Fe(III)-alginate aerogels. In PBS, the particles readily dissolved, and the rate was much faster in the case of the smaller beads. It is also worth to note that the kinetics was significantly different in the case of low $\mathrm{G}$ and high $\mathrm{G}$ samples, besides the size effect.

Drug release curves measured in $\mathrm{HCl}$ are shown in Figs. 7 and 8. Sink conditions did not apply in $\mathrm{HCl}$ as the total amount of loaded ibuprofen introduced into the release medium would almost saturate it upon dissolution. Under these conditions the aerogel formulations containing only the drug (S-LG-I, B-LG-I, S-HG-I and B-HG-I) did not display any difference in the kinetics of drug release. Neither the size of the beads, nor the composition of the backbone altered the rate significantly. The kinetics was also indifferent to the weight of loaded aerogel in the cuvette. This strongly suggest that the factor limiting the rate of drug release is the mass transport through the alginic acid layer present on the surface of the dissolving particles. It is noteworthy that incorporation of ascorbic acid slightly accelerated the release of ibuprofen in all samples. This proves that by in situ removing the crosslinking metal ion, the rate of drug release can be accelerated even in highly acidic media.

The release of ibuprofen was much faster in PBS than in $\mathrm{HCl}$ (Figs. 7 and 8). The experiments in PBS were run under sink conditions. Also, the dissolution of the Fe(III)-alginate matrices are much faster in this medium. Surprisingly, in the case of the high G samples loaded with ibuprofen alone (S-HG-I and B-HG-I) the rate of drug release dropped to near zero at reaching ca. $50 \%$ ibuprofen dissolution. Besides the above observations, it is noteworthy that the presence of ascorbic acid significantly increased both the rate and the extent of drug release in all samples. This effect was much more pronounced in PBS than in $\mathrm{HCl}$, which can be accounted for by the following. The aerogel beads take up more solvent and effectively swell in PBS, while this process is hindered in $\mathrm{HCl}$. The in situ reduction of the crosslinking Fe(III) is faster in a swollen hydrogel matrix, thus the accelerating effect is stronger in PBS. Another important observation is that the effect of ascorbic acid is more pronounced in the case of the high G samples. The explanation probably lies in the different hydration and dissolution properties of the different alginate backbones.

All release curves were compared to the Peppas and the Hopfenberg semi empirical models for drug release. In $\mathrm{HCl}$ release medium the shapes of the release curves approximately followed the Peppas model with $n$ between 0.40 and 0.44 . The curves recorded in PBS could not be successfully fitted with either release models.
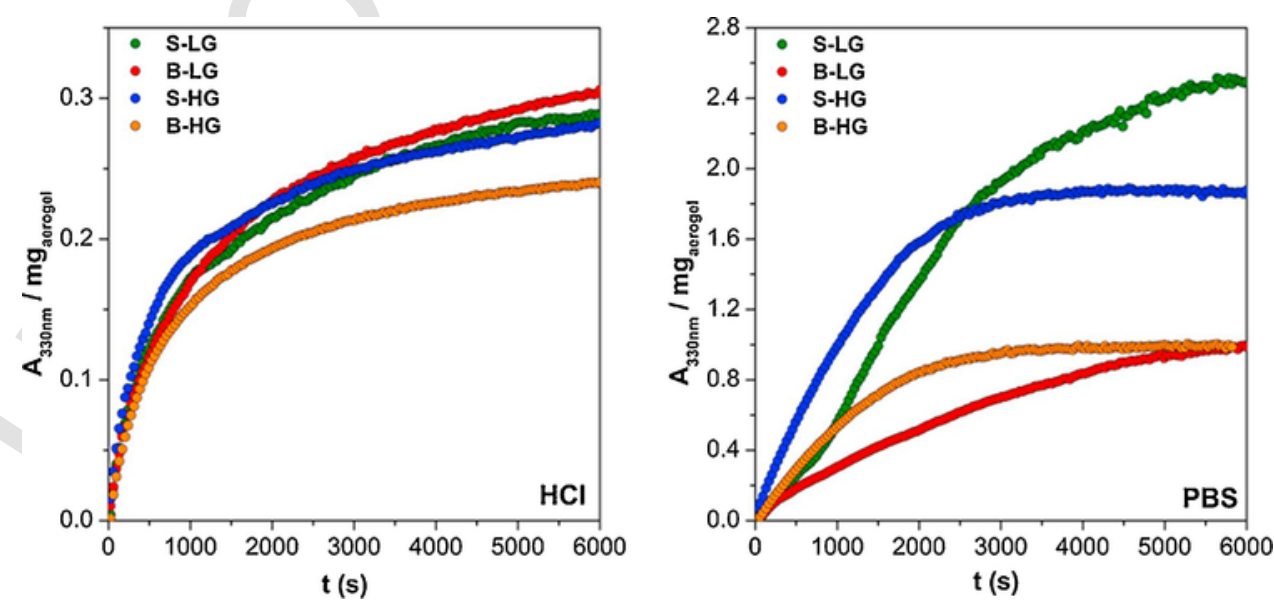

Fig. 6. Dissolution of pristine $\mathrm{Fe}(\mathrm{III})$-alginate aerogel samples at $\mathrm{pH}=2.0 \mathrm{in} \mathrm{HCl}$ (left) and at $\mathrm{pH}=7.4$ in $\mathrm{PBS}$ (right). Every 3rd point is shown for clarity. 

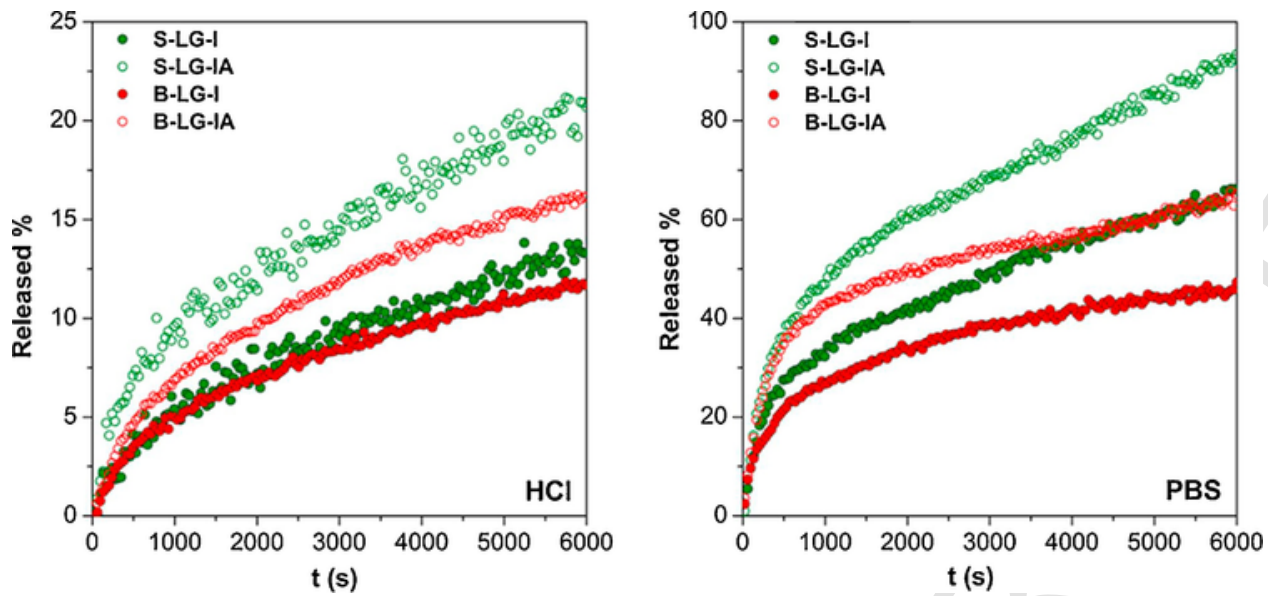

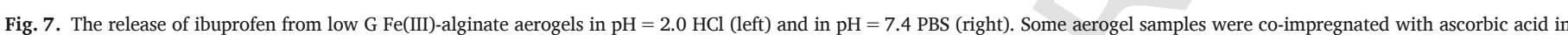
order to accelerate the release. Every 3rd point is shown for clarity.
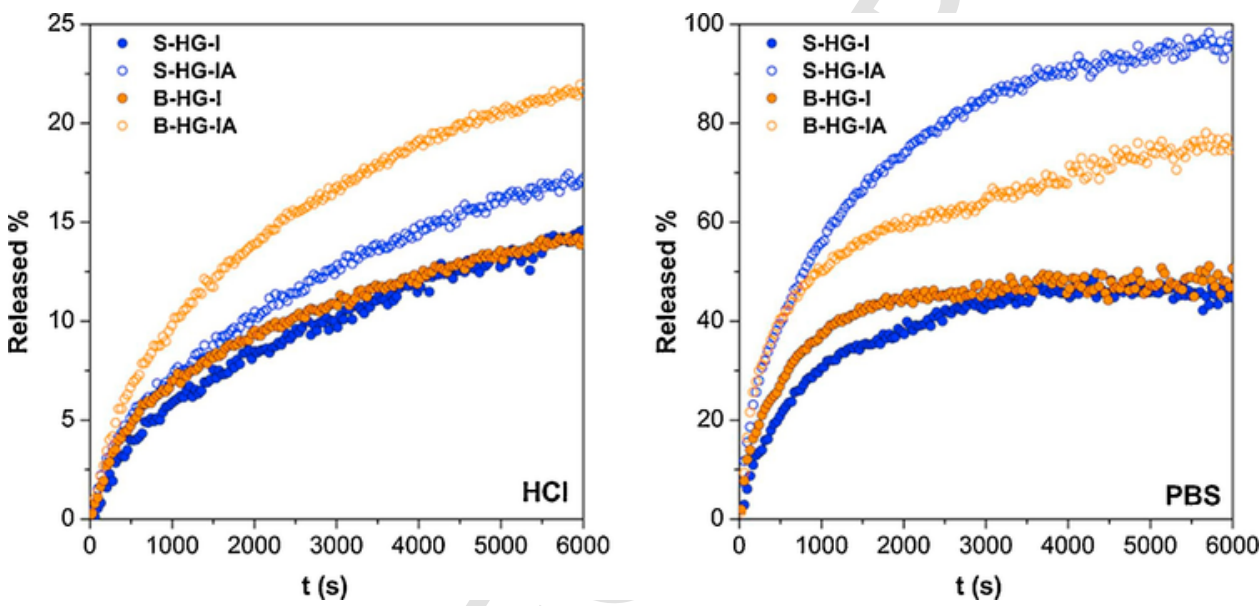

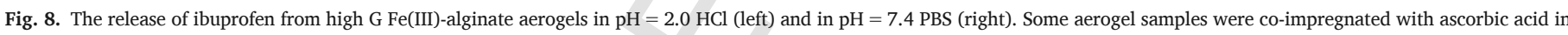
order to accelerate the release. Every 3rd point is shown for clarity.

As a summary, it is concluded that the size of the aerogel beads and the composition of the Fe(III)-alginate backbone have only minor effects on the rate of drug release. When the release curves shown in Figs. 7 and 8 are regrouped by the release media (Figs. S5 and S6 in the Supporting Information) the slight increase of the release rate with smaller bead size and higher $\mathrm{G}$ content can be observed. More importantly, the significant acceleration of the rate of drug release is achieved by incorporating ascorbic acid into the aerogel preparations in all cases.

\section{Conclusions}

It was shown that the release of the model drug ibuprofen from Fe(III)-alginate aerogel carriers is accelerated by incorporating ascorbic acid into the formulations. This was achieved by co-impregnating the aerogel samples with ibuprofen and ascorbic acid in supercritical $\mathrm{CO}_{2}$ using the adsorptive deposition (precipitation) technique. The in situ reduction of the crosslinking Fe(III) by ascorbic acid accelerates the erosion of the aerogel matrix upon dissolution, which in turn markedly increases the rate of the release of ibuprofen. Although this effect has previously been utilized for the development of redox responsive hydrogel-based delivery systems, this is the first time that such phenomenon has been demonstrated for solid aerogel-based formulations.

The effect of ascorbic acid is more pronounced at $\mathrm{pH}=7.4$ in PBS than at $\mathrm{pH}=2.0$ in $\mathrm{HCl}$, because the solvent uptake and the dissolu- tion of alginate beads are hindered in acidic media. The rate of drug release is further increased in the case of smaller carrier beads. The guluronic acid (G) content of the alginate backbone has only a slight effect on the carrier properties of the Fe(III)-alginate aerogel preparations, however the accelerating effect of ascorbic acid is stronger in high G samples.

\section{Acknowledgements}

The authors thank the Hungarian Science Foundation (OTKA: FK_17-124571 and PD-116224) for financial support. The project was supported by the ÚNKP-17-3 (P. Veres) and ÚNKP-17-4 (J. Kalmár) NEW NATIONAL EXCELLENCE PROGRAM of the Ministry of Human Capacities. The research was supported by the EU and co-financed by the European Regional Development Fund under the project GINOP-2.3.2-15-2016-00008. P. Gurikov and I. Smirnova are thankful to the DFG (projects SM 82/8-2 and >SM 82/8-3) for financial support.

\section{Appendix A. Supplementary data}

Supplementary data associated with this article can be found, in the online version, at https://doi.org/10.1016/j.carbpol.2018.01.098. 


\section{References}

Agulhon, P., Robitzer, M., David, L., Quignard, F., 2012. Structural regime identification in ionotropic alginate gels: Influence of the cation nature and alginate structure. Biomacromolecules 13 (1), 215-220.

Agulhon, P., Robitzer, M., Habas, J.P., Quignard, F., 2014. Influence of both cation and alginate nature on the rheological behavior of transition metal alginate gels. Carbohydrate Polymers 112, 525-531.

Alnaief, M., Alzaitoun, M.A., Garcia-Gonzalez, C.A., Smirnova, I., 2011. Preparation of biodegradable nanoporous microspherical aerogel based on alginate. Carbohydrate Polymers 84 (3), 1011-1018.

Bawa, P., Pillay, V., Choonara, Y.E., du Toit, L.C., 2009. Stimuli-responsive polymers and their applications in drug delivery. Biomedical Materials 4 (2), 022001.

Bruchet, M., Melman, A., 2015. Fabrication of patterned calcium cross-linked alginate hydrogel films and coatings through reductive cation exchange. Carbohydrate Polymers 131, 57-64.

Bruchet, M., Mendelson, N.L., Melman, A., 2013. Photochemical patterning of ionically cross-linked hydrogels. Processes 1 (2), 153-166.

Daemi, H., Barikani, M., 2012. Synthesis and characterization of calcium alginate nanoparticles, sodium homopolymannuronate salt and its calcium nanoparticles. Science Iran 19 (6), 2023-2028.

De Cicco, F., Russo, P., Reverchon, E., Garcia-Gonzalez, C.A., Aquino, R.P., Del Gaudio, P., 2016. Prilling and supercritical drying: A successful duo to produce core-shell polysaccharide aerogel beads for wound healing. Carbohydrate Polymers 147, 482-489.

Ditroi, T., Kalmar, J., Pino-Chamorro, J.A., Erdei, Z., Lente, G., Fabian, I., 2016. Construction of a multipurpose photochemical reactor with on-line spectrophotometric detection. Photochemical \& Photobiological Sciences 15 (4), 589-594.

Dong, Y.C., Dong, W.J., Cao, Y.N., Han, Z.B., Ding, Z.Z., 2011. Preparation and catalytic activity of Fe alginate gel beads for oxidative degradation of azo dyes under visible light irradiation. Catalysis Today 175 (1), 346-355.

Garcia-Gonzalez, C.A., Alnaief, M., Smirnova, I., 2011. Polysaccharide-based aerogels-promising biodegradable carriers for drug delivery systems. Carbohydrate Polymers 86 (4), 1425-1438.

Garcia-Gonzalez, C.A., Jin, M., Gerth, J., Alvarez-Lorenzo, C., Smirnova, I., 2015. Polysaccharide-based aerogel microspheres for oral drug delivery. Carbohydrate Polymers $117,797-806$.

Giammanco, G.E., Sosnofsky, C.T., Ostrowski, A.D., 2015. Light-responsive iron(III)-polysaccharide coordination hydrogels for controlled delivery. ACS Applied Materials \& Interfaces 7 (5), 3068-3076.

Glatter, O., Kratky, O. (Eds.), 1982. Small-angle X-ray scattering. Academic Press, New York.

Goncalves, V.S., Gurikov, P., Poejo, J., Matias, A.A., Heinrich, S., Duarte, C.M., Smirnova, I., 2016. Alginate-based hybrid aerogel microparticles for mucosal drug delivery. European Journal of Pharmaceutics and Biopharmaceutics 107, 160-170.

Gurikov, P., Smirnova, I., 2017. Amorphization of drugs by adsorptive precipitation from supercritical solutions: A review. Journal of Supercritical Fluids

Jánosi, A., 1986. Neue Aspekte der Phasenbestimmung an Festkörpern mittels Röntgenkleinwinkelstreuung. Zeitschrift für Physik B Condensed Matter 63 (3), 375-381.

Jin, Z., Guven, G., Bocharova, V., Halamek, J., Tokarev, I., Minko, S., Katz, E., 2012. Electrochemically controlled drug-mimicking protein release from iron-alginate thin-films associated with an electrode. ACS Applied Materials \& Interfaces 4 (1), 466-475.

Katz, E., Pingarron, J.M., Mailloux, S., Guz, N., Gamella, M., Melman, G., Melman, A., 2015. Substance release triggered by biomolecular signals in bioelectronic systems. The Journal of Physical Chemistry Letters 6 (8), 1340-1347.

Lee, B.-J., Min, G.-H., Kim, T.-W., 1996. Preparation andin vitro release of melatonin-loaded multivalent cationic alginate beads. Archives of Pharmacal Research 19 (4), 280.
Li, Y., Maciel, D., Rodrigues, J., Shi, X., Tomas, H., 2015. Biodegradable polymer nanogels for drug/nucleic acid delivery. Chemical Reviews 115 (16), 8564-8608.

Liu, H., Zhu, J.Y., Chai, X.S., 2011. In situ, rapid, and temporally resolved measurements of cellulase adsorption onto lignocellulosic substrates by UV-vis spectrophotometry. Langmuir 27 (1), 272-278.

Maciel, D., Figueira, P., Xiao, S., Hu, D., Shi, X., Rodrigues, J., Li, Y., 2013. Redox-responsive alginate nanogels with enhanced anticancer cytotoxicity. Biomacromolecules 14 (9), 3140-3146.

Mehling, T., Smirnova, I., Guenther, U., Neubert, R.H.H., 2009. Polysaccharide-based aerogels as drug carriers. Journal of Non-Crystalline Solids 355 (50-51), 2472-2479.

Narayanan, R.P., Melman, G., Letourneau, N.J., Mendelson, N.L., Melman, A., 2012. Photodegradable iron(III) cross-linked alginate gels. Biomacromolecules 13 (8), 2465-2471.

Pantic, M., Knez, Z., Novak, Z., 2016. Supercritical impregnation as a feasible technique for entrapment of fat-soluble vitamins into alginate aerogels. Journal of Non-Crystalline Solids 432, 519-526.

Porod, G., 1951. Die Röntgenkleinwinkelstreuung von dichtgepackten kolloiden Systemen. Colloid \& Polymer Science 124 (2), 83-114.

Quignard, F., Valentin, R., Di Renzo, F., 2008. Aerogel materials from marine polysaccharides. New Journal of Chemistry 32 (8), 1300-1310.

Robitzer, M., David, L., Rochas, C., Di Renzo, F., Quignard, F., 2008. Nanostructure of calcium alginate aerogels obtained from multistep solvent exchange route. Langmuir 24 (21), 12547-12552.

Robitzer, M., David, L., Rochas, C., Di Renzo, F., Quignard, F., 2008. Supercritically-dried alginate aerogels retain the fibrillar structure of the hydrogels. Macromolecular Symposia 273, 80-84, [Wiley Online Library.].

Rousseeuw, P.J., Verboven, S., 2002. Robust estimation in very small samples. Computational Statistics \& Data Analysis 40 (4), 741-758.

Smirnova, I., Gurikov, P., 2017. Aerogels in chemical engineering: strategies toward tailor-made aerogels. Annu Rev Chem Biomol Eng 8 (0), 307-334.

Smirnova, I., Mamic, J., Arlt, W., 2003. Adsorption of drugs on silica aerogels. Langmuir 19 (20), 8521-8525.

Sriamornsak, P., Thirawong, N., Korkerd, K., 2007. Swelling, erosion and release behavior of alginate-based matrix tablets. European Journal of Pharmaceutics and Biopharmaceutics 66 (3), 435-450.

Subrahmanyam, R., Gurikov, P., Dieringer, P., Sun, M., Smirnova, I., 2015. On the road to biopolymer aerogels - dealing with the solvent. Gels 1 (2), 291-313.

Tkalec, G., Kranvogl, R., Uzunalic, A.P., Knez, Z., Novak, Z., 2016. Optimisation of critical parameters during alginate aerogels' production. Journal of Non-Crystalline Solids $443,112-117$.

Ulker, Z., Erkey, C., 2014. A novel hybrid material: An inorganic silica aerogel core encapsulated with a tunable organic alginate aerogel layer. Rsc Advances 4 (107), 62362-62366.

van Hoogmoed, C.G., Busscher, H.J., de Vos, P., 2003. Fourier transform infrared spectroscopy studies of alginate-PLL capsules with varying compositions. Journal of Biomedical Materials Research Part A 67 (1), 172-178.

Veres, P., Keri, M., Banyai, I., Lazar, I., Fabian, I., Domingo, C., Kalmar, J., 2017. Mechanism of drug release from silica-gelatin aerogel-relationship between matrix structure and release kinetics. Colloids and Surfaces B-Biointerfaces 152, 229-237.

Veronovski, A., Novak, Z., Knez, Z., 2012. Synthesis and use of organic biodegradable aerogels as drug carriers. Journal of Biomaterials Science-Polymer Edition 23 (7), 873-886.

Veronovski, A., Knez, Z., Novak, Z., 2013. Preparation of multi-membrane alginate aerogels used for drug delivery. Journal of Supercritical Fluids 79, 209-215.

Wang, Q.Q., Zhu, J.Y., Hunt, C.G., Zhan, H.Y., 2012. Kinetics of adsorption, desorption, and re-adsorption of a commercial endoglucanase in lignocellulosic suspensions. Biotechnology and Bioengineering 109 (8), 1965-1975.

Zhao, S., Malfait, W.J., Guerrero Alburquerque, N., Koebel, M.M., Nystrom, G., 2018. Biopolymer aerogels: Chemistry, properties and applications. Angewandte Chemie International Edition In English 